\title{
Ambiguity, Money Transfers, and Endogenous Fluctuations
}

\author{
Eisei Ohtaki \\ Department of Economics, Kanagawa University, Kanagawa, Japan \\ Email: ohtaki@kanagawa-u.ac.jp
}

How to cite this paper: Ohtaki, E. (2021). Ambiguity, Money Transfers, and Endogenous Fluctuations. Theoretical Economics Letters, 11, 209-225.

https://doi.org/10.4236/tel.2021.112015

Received: January 29, 2021

Accepted: March 30, 2021

Published: April 2, 2021

Copyright $(\odot 2021$ by author(s) and Scientific Research Publishing Inc. This work is licensed under the Creative Commons Attribution International License (CC BY 4.0).

http://creativecommons.org/licenses/by/4.0/

\begin{abstract}
This article reexamines the implications of stochastic lump-sum money transfers in a monetary overlapping generations model, wherein agents are endowed with maxmin expected utility preferences. It is shown that: 1) there exists a continuum of stationary monetary equilibria, wherein a unique optimal one might exist, and 2) there exist innumerable nonstationary monetary equilibria, wherein the real money balance can move freely within a certain range.

\section{Keywords}

Lump-Sum Money Transfer, Indeterminacy, Endogenous Business Cycle, Ambiguity, Overlapping Generations Model
\end{abstract}

\section{Introduction}

In endogenous business cycle theory à la Grandmont $(1985,1986)$, it is well-known that lump-sum money transfer plays an important role in the stabilization of business cycles. However, it is also well-known that the introduction of lump-sum money transfer causes a decrease in economic welfare (Wallace, 1980). One might find in Brock and Scheinkman (1980), a way to overcome this trade-off between the stabilization of cycles and optimality. They considered lump-sum money transfers with the stochastic growth of money supply and showed that an increase in the mean of the rate of growth of the money supply induces a welfare loss and that an increase in the variance of the rate of growth of the money supply may cause an increase in welfare. By combining these results, it might be possible to observe that random lump-sum money transfers with a sufficiently high variance can attain simultaneously the stabilization of cycles and the mitigation of welfare loss. 
The aim of this article is to reexamine such a role of random lump-sum money transfers when the class of preferences is expanded beyond the standard subjective expected utility. Since Savage's (1954) pioneering work, most studies on economics under uncertainty have considered agents who choose their actions as if they maximize the expected utility with a single prior. Ellsberg's (1961) example, however, presented a situation in which agents might not assign a unique prior to uncertainty because of imprecise information. A representative model describing such a decision-making when information is imprecise is the class of maxmin expected utility (MEU) preferences, axiomatized by Schmeidler (1989) and Gilboa and Schmeidler (1989). Agents endowed with MEU preferences choose their actions as if they maximize the expected utility minimized over a set of priors. Now, a situation that agent's belief is summarized by a set of priors is called ambiguity.

Following Brock and Scheinkman (1980), this study considers an overlapping generations (OLG) model with random lump-sum money transfers, although agents are endowed with MEU preferences. This article contributes to the literature by making three main achievements. First, it is shown that a continuum of stationary monetary equilibria can exist, whereas the economy described by Brock and Scheinkman (1980) has a unique stationary monetary equilibrium. Second, this study finds that there might exist a unique golden rule optimal equilibrium in the continuum of stationary monetary equilibria. This is in sharp contrast to the well-known fact that the introduction of lump-sum money transfers induces a welfare loss ${ }^{1}$. Finally, it is shown that there exist innumerable nonstationary monetary equilibria, wherein the real money balance can move freely within an appropriate positive range. This might represent a remarkable difference from the existing results of Grandmont $(1985,1986)$. He argued that chaotic behaviors of equilibria can be observed when the nonlinearity derived from preferences is extremely high, and the introduction of lump-sum money transfers helps to stabilize cycles. In contrast, our result does not require high nonlinearity and the introduction of random lump-sum money transfers causes complex equilibrium dynamics.

This article also contributes to the ever-growing literature on the applications of ambiguity to various economic issues. In the last three decades, the implications of ambiguity have been explored in several economic environments. For example, Dow and Werlang (1992) and Epstein and Wang (1994) found asset price indeterminacy under ambiguity. Rigotti and Shannon (2012) found that ambiguity has less role in a canonical general equilibrium setting. Nishimura and Ozaki $(2004,2007)$ applied ambiguity to the search problem and the irreversible investments. Fukuda (2008) found a poverty trap in an OLG model with ambiguity. Guo and Yannelis (2021) applied ambiguity to implementation theory. However, few studies have applied ambiguity to monetary issues. In addition to the study by Ohtaki and Ozaki (2015), this study is one of the few scarce works ${ }^{1}$ See Wallace (1980), whereas his model is under certainty. 
that applied ambiguity to monetary theory.

The remainder of this paper is as follows. Section 2 presents our model, which is a variant of Brock and Scheinkman (1980). Section 3 provides main results. Section 4 contains some concluding remarks. The proofs of main results are relegated to the Appendix.

\section{The Model}

This paper considers a stationary, two-period, monetary overlapping generations model, wherein agents are endowed with the class of maxmin expected utility preferences. Uncertainty enters into the model through monetary shocks, described by stochastic money transfers.

\subsection{Physical Environment}

Time is discrete and runs from $t=1$ to infinity. In each period, there is a single perishable physical good, called the consumption good, and a single agent is born and lives for two periods. Thus, agents might be indexed by $t$, which is the period in which they are born. All agents are endowed with $w^{1}$ units of the consumption good in her first period of life and $w^{2}$ units in the second period, where $w^{1}>0$ and $w^{2} \geq 0$. Agent $t$ consumes $c_{t}^{1}$ and $c_{t+1}^{2}$ units of the consumption good in her first and second periods, respectively. Agent $t$ is assumed to rank her consumption plans $\left(c_{t}^{1}, c_{t+1}^{2}\right)$ according to a lifetime utility function $u: \mathfrak{R}_{+}^{2} \rightarrow \mathfrak{R}$. We assume throughout, unless specified otherwise, that $u$ is timeseparable and there exist strictly increasing, strictly concave, and continuously differentiable real-valued functions $v_{1}$ and $v_{2}$ on $\mathfrak{R}_{+}$such that

$$
\left(\forall\left(c^{1}, c^{2}\right) \in \mathfrak{R}_{+}^{2}\right) u\left(c^{1}, c^{2}\right)=v_{1}\left(c^{1}\right)+v_{2}\left(c^{2}\right)
$$

and $\lim _{x \downarrow 0} v_{i}^{\prime}(x)=\infty$ for each $i=1,2^{2}$.

In period 1, there also exists a one-period-lived agent, called the initial old. The agent aims to maximize her consumption, $c_{1}^{2} \geq 0$, in period 1 .

\subsection{Stochastic Money Transfer}

This study considers stochastic money transfers to old agents as monetary shocks. Let $Z=\left\{z^{1}, \cdots, Z^{S}\right\}$ be a nonempty finite set of $\mathfrak{R}_{++}$. We regard each element $Z \in Z$ as a gross rate of growth of money stock realized in each single period and also call it a state. It is assumed that the state in each period realizes before the new agent in that period enters the economy. Let $z_{0} \in Z$ be a given initial state (in period 0 , which is implicitly defined). The date-event tree, $\Gamma$, is defined as follows: 1) the root of the tree is $Z_{0} ; 2$ ) the set of nodes at date $t \geq 1$ is denoted by $\Sigma_{t}$, where we set $Z_{1}:=\left\{z_{0}\right\} \times Z$ and, iteratively, $\Sigma_{t}:=\Sigma_{t-1} \times Z$ for $t \geq 2$; and 3) $\Sigma=\bigcup_{t \geq 1} \Sigma_{t}$ and $\Gamma:=\left\{z_{0}\right\} \cup \Sigma$. Note that, for any date-event ${ }^{2} \mathrm{We}$ assume strict concavity and the boundary conditions on $v_{1}$ and $v_{2}$ in order to provide a sharper argument. To tell the truth, however, we can replace strict concavity of $v_{1}$ with concavity and can remove the boundary conditions on $v_{1}$ and $v_{2}$, provided that the possible money growth rates are given appropriately. 
$\sigma \in \Gamma$, there is a unique predecessor node, denoted by $\sigma_{-}$. Also, let $\Delta_{Z}$ be the set of probability measures on $Z$ and $\Delta_{Z}^{\circ}:=\Delta_{Z} \cap \Re_{++}^{Z}$.

Let $M_{0}>0$ be the initial stock of money (in the period 0 ). For any $\sigma^{t}=\left(z_{0}, z_{1}, \cdots, z_{t}\right) \in \Sigma_{t}$, the stock of money at date-event $\sigma^{t}$ is denoted by $M_{t} \equiv M\left(\sigma^{t}\right):=z_{t} \times \cdots \times z_{1} M_{0}$. At date-event $\sigma^{t+1} \in \Sigma_{t+1}$, the government issues $T_{t+1} \equiv T\left(\sigma^{t+1}\right):=M\left(\sigma^{t+1}\right)-M\left(\sigma_{-}^{t+1}\right)$ units of new money and gives it to the old agent at the date-event as lump-sum transfers. The period 1 money stock $M_{1} \equiv M\left(z_{0}, z_{1}\right)$ is held by the initial old. We will denote by $p(\sigma)$ and $q(\sigma)$ the real price of money and the (per-capita) real money balance at a date-event $\sigma \in \Sigma$, respectively. Of course, $q(\sigma)=p(\sigma) M(\sigma)$ for each date-event $\sigma \in \Sigma$, so that, the real price of money has a one-to-one relation to the real money balance.

Remark that states in our model are extrinsic in the sense that they do not affect the initial endowment streams $\left(w^{1}, w^{2}\right)$ nor the lifetime utility function $u$. Therefore, one might be able to interpret states as extrinsic uncertainty or sunspots. Our monetary shocks can also be interpreted, for examples, as a nondeterministic relation between monetary bases and money stocks or as unanticipated monetary policies ${ }^{3}$.

\subsection{MEU and Equilibrium}

Throughout the remainder of this paper, we assume that each agent's belief on the realization of states in the second period of her life is represented by a nonempty, compact, and convex subset of $\Delta_{Z}^{\circ}$, denoted by $\mathcal{P}$, which is independent of time and current and past realizations of states. Further, for any $c=\left(c^{1}, c^{2}\right) \in \mathfrak{R}_{+} \times \mathfrak{R}_{+}^{Z}$, each agent's preference is assumed to be represented by the maxmin expected utility

$$
U(c):=\min _{\pi \in \mathcal{P}} \sum_{z^{\prime} \in Z} u\left(c^{1}, c^{2}\left(z^{\prime}\right)\right) \pi_{z^{\prime}}=v_{1}\left(c^{1}\right)+\min _{\pi \in \mathcal{P}} \sum_{z^{\prime} \in Z} v_{2}\left(c^{2}\left(z^{\prime}\right)\right) \pi_{z^{\prime}}
$$

Note that $U(c)$ is strictly concave because of strict concavity of $v_{1}$ and $v_{2}$. Also assume throughout, unless specified otherwise, that $\max _{\pi \in \mathcal{P}} \sum_{z \in Z} z^{-1} \pi_{z}>\min _{\pi \in \mathcal{P}} \sum_{z \in Z} z^{-1} \pi_{z}$.

Now, we are ready to define an equilibrium. Because the real price of money and the real money balance at each date-event have the one-to-one relation, this study defines an equilibrium in terms of real money balances, not real prices of money. An equilibrium is a process of real money balances, $q^{e}: \Sigma \rightarrow \mathfrak{R}_{+}$, with some process of money holdings $m^{e}: \Sigma \rightarrow \mathfrak{R}$ such that: given any $\left.\sigma \in \Sigma, 1\right)$ $m^{e}(\sigma)$ maximizes $U\left(c^{1}(\sigma),\left(c^{2}\left(\sigma, z^{\prime}\right)\right)_{z^{\prime} \in Z}\right)$ subject to sequential budget constraints

$$
c^{1}(\sigma)=w^{1}-\frac{q^{e}(\sigma)}{M(\sigma)} m \text { and }\left(\forall z^{\prime} \in Z\right) c^{2}\left(\sigma, z^{\prime}\right)=w^{2}+\frac{q^{e}\left(\sigma, z^{\prime}\right)}{M\left(\sigma, z^{\prime}\right)}\left(m+T\left(\sigma, z^{\prime}\right)\right)
$$

and 2) $m^{e}(\sigma)=M(\sigma)$. This is a standard definition of equilibrium: condition ${ }^{3}$ Sunspot money transfers as in our model is also considered in the previous studies such as Brock and Scheinkman (1980). 
(1) is the MEU-maximizing problem with sequential budget constraints and condition (2) is the market-clearing condition for money. An equilibrium $q^{e}$ is

- Stationaryif there is some $q^{\text {se }} \in \mathfrak{R}_{+}^{Z}$ such that $q^{e}(\sigma, z)=q_{z}^{\text {se }}$ for any $\sigma \in \Gamma$ and any $z \in Z$;

- Deterministic if there is a nonnegative sequence $\left\{\bar{q}_{t}^{e}\right\}_{t=1}^{\infty}$ such that $q^{e}\left(\sigma^{t}\right)=\bar{q}_{t}^{e}$ for any $t \geq 1$ and any $\sigma^{t} \in \Sigma_{t}$; and

- Monetary if it is positive-valued.

In order to close this section, we provide two remarks. Remark first that our $q^{e}$ is real money balances, not real prices of money. If one wish to obtain real prices of money, let $p^{e}(\sigma):=q^{e}(\sigma) / M(\sigma)$ for each $\sigma \in \Sigma$. At an equilibrium $q^{e}$, we can obtain that, for each $\sigma \in \Sigma$ and each $z^{\prime} \in Z$,

$$
\frac{p^{e}\left(\sigma, z^{\prime}\right)}{p^{e}(\sigma)}=\frac{p^{e}\left(\sigma, z^{\prime}\right) M\left(\sigma, z^{\prime}\right)}{p^{e}(\sigma) M\left(\sigma, z^{\prime}\right)}=\frac{q^{e}\left(\sigma, z^{\prime}\right)}{z^{\prime} q^{e}(\sigma)}
$$

as the real rate of return of money, which is not necessarily equal to one due to stochastic growth of the money stock, and

$$
p^{e}\left(\sigma, z^{\prime}\right) T\left(\sigma, z^{\prime}\right)=p^{e}\left(\sigma, z^{\prime}\right)\left[M\left(\sigma, z^{\prime}\right)-M(\sigma)\right]=\frac{z^{\prime}-1}{z^{\prime}} q^{e}\left(\sigma, z^{\prime}\right)
$$

as the real amount of money transfers. Also remark that, at an equilibrium $q^{e}$, the market-clearing conditions for the consumption good holds at each dateevent $\sigma \in \Sigma$. In fact, given an equilibrium $q^{e}$, it holds that

$$
\begin{aligned}
& c^{1}(\sigma)+c^{2}(\sigma) \\
& =\left[w^{1}-\frac{q^{e}(\sigma)}{M(\sigma)} m^{e}(\sigma)\right]+\left[w^{2}+\frac{q^{e}(\sigma)}{M(\sigma)}\left(m^{e}\left(\sigma_{-}\right)+T(\sigma)\right)\right] \\
& =w^{1}+w^{2},
\end{aligned}
$$

where the first equality follows from the sequential budget constraints and the second equality follows from the money market clearing condition. This is no doubt the market clearing condition for the consumption good at date-event $\sigma \in \Sigma^{4}$. In other words, each allocation associated with an equilibrium is always feasible. One might note that lump-sum money transfers in our model do not affect the feasibility condition on equilibrium allocation.

\section{Main Results}

\subsection{Characterization of Equilibrium}

Our first task is to characterize a monetary equilibrium. When agents' preferences are represented by standard expected utility functions, a monetary equilibrium is characterized by a system of difference equations $s^{5}$. However, because the class of MEU preferences are not smooth at some points ${ }^{6}$, a monetary equili-

${ }^{4}$ Note that $m^{e}\left(\sigma_{-}^{1}\right) \equiv m^{e}\left(z_{0}\right)=M_{1}$.

${ }^{5}$ See for example Ohtaki $(2011,2015)$.

${ }^{6}$ To be more precise, indifference hyperplanes have kinks at consumption plans $\left(c^{1}, c^{2}\right) \in \mathfrak{R}_{+}^{1+s}$ such that $c^{2}(z)=c^{2}\left(z^{\prime}\right)$ for $z, z^{\prime} \in Z$. 
brium is characterized by a system of difference inclusions.

Proposition 1. A stochastic process $q^{e}: \Sigma \rightarrow \mathfrak{R}$ such that $\left.q^{e}(\sigma) \in\right] 0, w^{1}[$ for each $\sigma \in \Sigma$ is a monetary equilibrium if and only if

$$
q^{e}(\sigma) v_{1}^{\prime}\left(w^{1}-q^{e}(\sigma)\right) \in\left\{\sum_{z^{\prime} \in Z} \frac{1}{z^{\prime}} q^{e}\left(\sigma, z^{\prime}\right) v_{2}^{\prime}\left(w^{2}+q^{e}\left(\sigma, z^{\prime}\right)\right) \pi_{z^{\prime}} \mid \pi \in \mathcal{B}_{\sigma}\right\}
$$

for each $\sigma \in \Sigma$, where

$$
\mathcal{B}_{\sigma}:=\min _{\pi \in \mathcal{P}} \sum_{z^{\prime} \in Z} v_{2}\left(w^{2}+q^{e}\left(\sigma, z^{\prime}\right)\right) \pi_{z^{\prime}}
$$

which is the set of probability measures minimizing the second-period expected utility over $\mathcal{P}$.

As an immediate corollary, we can find that there is no stationary monetary equilibrium $q^{\text {se }}=\left(q_{z}^{s e}\right)_{z \in Z}$ such that $q_{z}^{s e} \neq q_{z^{\prime}}^{s e}$ for each $z, z^{\prime} \in Z$. Suppose the contrary that there is some $q^{\text {se }}$ such that $w^{2}+q_{z}^{s e} \neq w^{2}+q_{z^{\prime}}^{\text {se }}$ for each $z, z^{\prime} \in Z$. This implies the existence of some $\pi \in \mathcal{P}$ such that $B_{\sigma}=\{\pi\}$ for each $\sigma \in \Sigma$. The $q^{\text {se }}$ is now characterized by

$$
(\forall z \in Z) \quad q_{z}^{s e} v_{1}^{\prime}\left(w^{1}-q_{z}^{s e}\right)=\sum_{z^{\prime} \in Z} \frac{1}{z^{\prime}} q_{z^{\prime}}^{s e} v_{2}^{\prime}\left(w^{2}+q_{z^{\prime}}^{s e}\right) \pi_{z^{\prime}}
$$

Because $q v_{1}^{\prime}\left(w^{1}-q\right)$ is strictly increasing in $q$ and the right-hand side of the above equation is independent of $z, q_{z}^{\text {se }}$ must be independent of $z$, which contradicts the hypothesis that $q_{z}^{s e} \neq q_{z^{\prime}}^{\text {se }}$ for each $z, z^{\prime} \in Z$. Therefore, there is no stationary monetary equilibrium $q^{\text {se }}$ such that $q_{z}^{s e} \neq q_{z^{\prime}}^{\text {se }}$ for each $z, z^{\prime} \in Z$.

\subsection{Existence and Indeterminacy of Stationary Monetary Equilibrium}

As mentioned in the previous subsection, there is no stationary monetary equilibrium $q^{\text {se }}$ such that $q_{z}^{s e} \neq q_{z^{\prime}}^{\text {se }}$ for each $z, z^{\prime} \in Z$. Of course, there might exist a stationary monetary equilibrium $q^{\text {se }}$ such that $q_{z}^{s e}=q_{z^{\prime}}^{s e} \neq q_{z^{\prime \prime}}^{\text {se }}$ for some distinct $z, z^{\prime}, z^{\prime \prime} \in Z$. In order to obtain shaper results, however, we concentrate our attention on a stationary deterministic monetary equilibrium.

At a stationary deterministic monetary equilibrium, it follows that $\mathcal{B}_{\sigma}$ in Proposition 1 is equal to $\mathcal{P}$ because $v_{2}\left(w^{2}+q^{e}\left(\sigma, z^{\prime}\right)\right)$ is constant over $Z$. Therefore, as a corollary of Proposition 1, a stationary deterministic monetary equilibrium $q^{\text {se }}$ can be identified with the real number $\bar{q}^{\text {se }} \in\left(0, w^{1}\right)$, which is a solution of the inclusion

$$
\bar{q}^{s e} v_{1}^{\prime}\left(w^{1}-\bar{q}^{s e}\right) \in\left\{\sum_{z^{\prime} \in Z} \frac{1}{z^{\prime}} \bar{q}^{s e} v_{2}^{\prime}\left(w^{2}+\bar{q}^{s e}\right) \pi_{z^{\prime}} \mid \pi \in \mathcal{P}\right\}
$$

or equivalently

$$
\underline{\zeta}:=\min _{\pi \in \mathcal{P}} \sum_{z \in Z} z^{-1} \pi_{z} \leq \frac{v_{1}^{\prime}\left(w^{1}-\bar{q}^{s e}\right)}{v_{2}^{\prime}\left(w^{2}+\bar{q}^{s e}\right)} \leq \max _{\pi \in \mathcal{P}} \sum_{z \in Z} z^{-1} \pi_{z}=: \bar{\zeta} .
$$

Examining this inequality, we can obtain the following result. 
Proposition 2. For each $\zeta$ such that $\underline{\zeta} \leq \zeta \leq \bar{\zeta}$, if $\zeta>v_{1}^{\prime}\left(w^{1}\right) / v_{2}^{\prime}\left(w^{2}\right)$, each $\bar{q}^{\text {se }} \in \bar{Q}(\zeta)$ is a stationary deterministic monetary equilibrium, where $\bar{Q}(\zeta):=[\bar{q}(\zeta), \bar{q}(\bar{\zeta})]$ and $\bar{q}(x)$ is a solution of the equation that $v_{1}^{\prime}\left(w^{1}-q\right)=x v_{2}^{\prime}\left(w^{2}+q\right)$ with respect to $q$.

This proposition says that every element of $\bar{Q}(\zeta)$ can construct a stationary deterministic monetary equilibrium, and therefore, the stationary deterministic monetary equilibrium is indeterminate. As argued in Subsection 3.4, agents partially sacrifice consumption smoothing to avoid ambiguity. In the OLG framework, it enlarges the intergenerational transfers supported at monetary equilibrium. This is the main reason of indeterminacy of stationary monetary equilibrium.

Note that, if $\underline{\zeta}>v_{1}^{\prime}\left(w^{1}\right) / v_{2}^{\prime}\left(w^{2}\right)$, then $\bar{Q}(\underline{\zeta})$ is the largest set of stationary deterministic monetary equilibria, i.e., there is no stationary deterministic monetary equilibrium $q$ such that $q \in\left(0, w^{1}\right] \backslash \bar{Q}(\underline{\zeta})$. Suppose the contrary. There are two possible cases: 1) $q \in(0, \bar{q}(\underline{\zeta}))$ and 2) $q \in\left(\bar{q}(\bar{\zeta}), w^{1}\right]$. In case (1), it follows from strict concavity of $v_{1}$ and $v_{2}$ that $v_{1}^{\prime}\left(w^{1}-q\right)<v_{2}^{\prime}\left(w^{2}+q\right) \underline{\zeta}$. In case (2), on the other hand, it follows from strict concavity of $v_{1}$ and $v_{2}$ that $v_{1}^{\prime}\left(w^{1}-q\right)<v_{2}^{\prime}\left(w^{2}+q\right) \bar{\zeta}$. In any cases, $q$ contradicts the definition of stationary deterministic monetary equilibrium (and Proposition 1). Therefore, if $\underline{\zeta}>v_{1}^{\prime}\left(w^{1}\right) / v_{2}^{\prime}\left(w^{2}\right)$, we can conclude that $\bar{Q}(\underline{\zeta})$ is the largest set of stationary deterministic monetary equilibria.

\subsection{Optimality of Stationary Monetary Equilibrium}

We then examine the existence of optimal monetary equilibrium. Here, optimality is (ex-post) Pareto optimality. Some of this reason is the economy is deterministic, except for monetary policy ${ }^{7}$. An allocation $\left\{c_{t}^{1}, c_{t}^{2}\right\}_{t \geq 1}$ satisfying that $c_{t}^{1}+c_{t}^{2}=w^{1}+w^{2}$ for all $t \geq 1$ is Pareto optimal if there is no other allocation $\left\{b_{t}^{1}, b_{t}^{2}\right\}_{t \geq 1}$ satisfying that $b_{t}^{1}+b_{t}^{2}=w^{1}+w^{2}$ for all $t \geq 1$ such that $b_{1}^{2} \geq c_{1}^{2}$ and

$u\left(b_{t}^{1}, b_{t+1}^{2}\right) \geq u\left(c_{t}^{1}, c_{t+1}^{2}\right)$ for all $t \geq 1$ with strict inequality somewhere. Also, an allocation $\left\{c_{t}^{1}, c_{t}^{2}\right\}_{t \geq 1}$ satisfying that $c_{t}^{1}+c_{t}^{2}=w^{1}+w^{2}$ for all $t \geq 1$ is golden rule optimal if $c_{t}^{1}=c^{* 1}$ and $c_{t}^{2}=c^{* 2}$ for all $t \geq 1$, where $\left(c^{* 1}, c^{* 2}\right)$ is a unique solution of the problem: $\max _{c^{1}, c^{2}} u\left(c^{1}, c^{2}\right)$ subject to $c^{1}+c^{2}=w^{1}+w^{2}$. Note that an interior golden rule optimal allocation $\left(c^{* 1}, c^{* 2}\right)$ is completely characterized by $c^{* 1}+c^{* 2}=w^{1}+w^{2}$ and $v_{1}^{\prime}\left(c^{* 1}\right) / v_{2}^{\prime}\left(c^{* 2}\right)=1$. Also note that any golden rule optimal allocation is Pareto optimal, given current assumptions.

Proposition 3. Assume that $v_{1}^{\prime}\left(w^{1}\right) / v_{2}^{\prime}\left(w^{2}\right)<1$. Then, there is a stationary deterministic monetary equilibrium, allocation of which is golden rule optimal, if and only if $\underline{\zeta} \leq 1 \leq \bar{\zeta}$. Furthermore, the equilibrium is unique.

${ }^{7}$ For (ex-ante) optimality in stochastic environments, see Chattopadhyay and Gottardi (1999) and Ohtaki (2013) for example.

${ }^{8}$ In other words, Pareto optimality cares welfare of all generations including the initial old, whereas golden rule optimality does welfare of all newly born agents only. Interested readers might find a more theoretical relationship between these two criteria on optimality in Ohtaki (2013). 
If $\mathcal{P}$ is a singleton with the unique element $\pi$, the last condition degenerates into $\sum_{z \in Z} z^{-1} \pi_{z}=1$. On the other hand, in the presence of ambiguity, we do not necessarily require that $\sum_{z \in Z} z^{-1} \pi_{z}=1$ to obtain the optimal monetary equilibrium.

Note that, as shown in Proposition 2, there might be a continuum of stationary monetary equilibria. By Proposition 3, however, the golden rule optimal equilibrium, if any, is unique. As a corollary of Proposition 3, therefore, we can say that the golden rule optimal equilibrium is measure zero in the space of stationary monetary equilibria.

\subsection{Complexity of Deterministic Monetary Equilibria}

It is well-known that, in a canonical pure-endowment OLG model with a twoperiod-lived agent per generation, stationary monetary equilibrium is unstable and each monetary equilibrium with a sufficiently low initial real money balance converges to the nonmonetary one. Here, we reexamine this observation in the present framework.

As a corollary of Proposition 1, a deterministic monetary equilibrium can be identified with a positive sequence $\left(\bar{q}_{t}^{e}\right)_{t=1}^{\infty}$ such that, for each $t \geq 1$,

$$
\frac{\bar{q}_{t+1}^{e}}{\bar{q}_{t}^{e}} \underline{\zeta} \leq \frac{v_{1}^{\prime}\left(w^{1}-\bar{q}_{t}^{e}\right)}{v_{2}^{\prime}\left(w^{2}+\bar{q}_{t}^{e}\right)} \leq \frac{\bar{q}_{t+1}^{e}}{\bar{q}_{t}^{e}} \bar{\zeta},
$$

where $\underline{\zeta}$ and $\bar{\zeta}$ are defined as in Equation (1). This can be interpreted as a requirement that, in a deterministic monetary equilibrium, the marginal rate of substitution at the equilibrium allocation lies on the range of inflation-adjusted deflation rates. Moreover, we might be able to say that this is a trade-off between uncertainty aversion and consumption smoothing. That is, Equation (2) says that agents avoid uncertainty by partially sacrificing consumption smoothing.

We then study nonstationary deterministic monetary equilibria, which is a positive sequence $\left(\bar{q}_{t}^{e}\right)_{t=1}^{\infty}$ satisfying Equation (2). Throughout the rest of this subsection, let $\zeta$ be an arbitrary real number belonging to $[\underline{\zeta}, \bar{\zeta}]$ and assume that $\zeta>v_{1}^{\prime}\left(w^{1}\right) / v_{2}^{\prime}\left(w^{2}\right)$ and that $\bar{q}(\zeta)<\bar{q}(\bar{\zeta})$. Also let $\bar{q}^{*}$ and $\bar{q}_{*}$ be arbitrary elements of the set of stationary deterministic monetary equilibrium, $\bar{Q}(\zeta)$, such that $\bar{q}_{*}<\bar{q}^{*}$. Because $\bar{q}(\zeta)<\bar{q}(\bar{\zeta})$, the existence of such $\bar{q}^{*}$ and $\bar{q}_{*}$ is guaranteed. Define $\bar{Q}^{*}:=\left[\bar{q}_{*}, \bar{q}^{*}\right]$. We can now describe the first result of complex dynamics on equilibrium passes.

Proposition 4. If $-q v_{2}^{\prime \prime}\left(w^{2}+q\right) / v_{2}^{\prime}\left(w^{2}+q\right) \geq 1$ for all $q \in \bar{Q}^{*}$, then every sequence $\left(\bar{q}_{t}^{e}\right)_{t=1}^{\infty}$ such that $q_{t}^{e} \in \bar{Q}^{*}$ for each $t \geq 1$ is a deterministic monetary equilibrium.

Note that the condition that $-q v_{2}^{\prime \prime}\left(w^{2}+q\right) / v_{2}^{\prime}\left(w^{2}+q\right) \geq 1$ for all $q \in \bar{Q}^{*}$ holds, for example, if the index of relative risk aversion of $v_{2}$ is greater than unity and the initial endowment in the second period is sufficiently small. Actually, when $w^{2}=0$, the condition exactly holds if the index of relative risk aversion of $v_{2}$ is greater than of equal to unity. 
We next turn to the case that $-q v_{2}^{\prime \prime}\left(w^{2}+q\right) / v_{2}^{\prime}\left(w^{2}+q\right) \leq 1$.

Proposition 5. If $-q v_{2}^{\prime \prime}\left(w^{2}+q\right) / v_{2}^{\prime}\left(w^{2}+q\right) \leq 1$ for all $q \in \bar{Q}^{*}$ and

$$
\bar{q}^{*} v_{2}^{\prime}\left(w^{2}+\bar{q}^{*}\right) \underline{\zeta}<\bar{q}^{*} v_{2}^{\prime}\left(w^{2}+\bar{q}_{*}\right) \bar{\zeta}
$$

then $\bar{q}_{*} \leq \bar{q}_{* *}<\bar{q}^{* *} \leq \bar{q}^{*}$ and every sequence $\left(\bar{q}_{t}^{e}\right)_{t=1}^{\infty}$ such that $q_{t}^{e} \in\left[\bar{q}_{* *}, \bar{q}^{* *}\right]$ for each $t \geq 1$ is a deterministic monetary equilibrium, where $\bar{q}^{* *}$ and $\bar{q}_{* *}$ are solutions of equations $\bar{q}^{* *} v_{1}^{\prime}\left(w^{1}-\bar{q}^{* *}\right)=\bar{q}_{*} v_{2}^{\prime}\left(w^{2}+\bar{q}_{*}\right) \bar{\zeta}$ and $\bar{q}_{* *} v_{1}^{\prime}\left(w^{1}-\bar{q}_{* *}\right)=\bar{q}^{*} v_{2}^{\prime}\left(w^{2}+\bar{q}^{*}\right) \underline{\zeta}$, respectively.

Note that the condition that $-q v_{2}^{\prime \prime}\left(w^{2}+q\right) / v_{2}^{\prime}\left(w^{2}+q\right) \leq 1$ for all $q \in \bar{Q}^{*}$ holds, for example, if the index of relative risk aversion of $v_{2}$ is less than or equal to unity, i.e., $-x v_{2}^{\prime \prime}(x) / v_{2}^{\prime}(x) \leq 1$ for each $x>0$. In order to verify this, let $\psi(q):=q v_{2}^{\prime}\left(w^{2}+q\right)$ for $q>0$. Then, if $-x v_{2}^{\prime \prime}(x) / v_{2}^{\prime}(x) \leq 1$ for each $x>0$, $\psi^{\prime}(q)=v_{2}^{\prime}\left(w^{2}+q\right)+q v_{2}^{\prime \prime}\left(w^{2}+q\right) \geq v_{2}^{\prime}\left(w^{2}+q\right)+\left(w^{2}+q\right) v_{2}^{\prime \prime}\left(w^{2}+q\right) \geq 0$, which implies that $-q v_{2}^{\prime \prime}\left(w^{2}+q\right) / v_{2}^{\prime}\left(w^{2}+q\right) \leq 1$ for each $q \geq 0$.

Propositions 4 and 5 imply that, for any utility index functions $v_{1}$ and $v_{2}$, there might exist innumerable nonstationary monetary equilibria, wherein the real money balance can move freely within a certain range of positive numbers. This is far from the existing results in endogenous business cycle theory, in which the business cycles are explained by chaos theory.

\section{Concluding Remarks}

This article has explored the implications of stochastic lump-sum money transfers in a monetary OLG, wherein agents are endowed with MEU preferences. It has been shown that the deterministic equilibrium is characterized by a difference inclusion, not an equation, and stationary deterministic monetary equilibrium is indeterminate. Among such equilibria, it has been shown that an optimal one may exist. Furthermore, we have presented several conditions, under which we can observe innumerable nonstationary monetary equilibria, wherein the real money balance moves freely within a certain range of positive numbers. The last result is a remarkable difference from the existing literature such as Grandmont $(1985,1986)$, which have argued that endogenous business cycles require high nonlinearity to the model. In this sense, the last result represents a new mechanism of endogenous business cycles. It is a future work whether our mechanism of endogenous fluctuations can be observed in more general model with, for example, intertemporal production technology.

Remark that the stochastic growth of money in this study is as sunspots in the sense that it does not affect endowments nor preferences. Such money creation may be interpreted as a situation in which the monetary authority determines a policy based on false information. Our last result signifies that such decisions, based on false information, cause endogenous fluctuations.

\section{Acknowledgements}

The author thanks to two anonymous referees and Professors Yasuo Maeda, 
Shuhei Shiozawa, and Hiroyuki Ozaki for their helpful comments.

\section{Conflicts of Interest}

The author declares no conflicts of interest regarding the publication of this paper.

\section{References}

Brock, W. A., \& Scheinkman, J. A. (1980). Some Remarks on Monetary Policy in an Overlapping Generations Model. In J. H. Kareken and N. Wallace (Ed.) Models of Monetary Economies (pp. 211-232). Minneapolis, MN: Federal Reserve Bank of Minneapolis.

Chattopadhyay, S., \& Gottardi, P. (1999). Stochastic OLG Models, Market Structure, and Optimality. Journal of Economic Theory, 89, 21-67.

https://doi.org/10.1006/jeth.1999.2543

Dow, J., \& Werlang, S. R. C. (1992). Uncertainty Aversion, Risk Aversion, and the Optimal Choice of Portfolio. Econometrica, 60, 197-204. https://doi.org/10.2307/2951685

Ellsberg, D. (1961). Risk, Ambiguity, and the Savage Axioms. Quarterly Journal of Economics, 75, 643-669. https://doi.org/10.2307/1884324

Epstein, L. G., \& Wang, T. (1994). Intertemporal Asset Pricing under Knightian Uncertainty. Econometrica, 62, 283-322. https://doi.org/10.2307/2951614

Fukuda, S. I. (2008). Knightian Uncertainty and Poverty Trap in a Model of Economic Growth. Review of Economic Dynamics, 11, 652-663.

https://doi.org/10.1016/j.red.2007.11.001

Gilboa, I., \& Schmeidler, D. (1989). Maxmin Expected Utility with Non-Unique Prior. Journal of Mathematical Economics, 18, 141-153. https://doi.org/10.1016/0304-4068(89)90018-9

Grandmont, J. M. (1985). On Endogenous Competitive Business Cycles. Econometrica, 53, 995-1045. https://doi.org/10.2307/1911010

Grandmont, J. M. (1986). Stabilizing Competitive Business Cycles. Journal of Economic Theory, 40, 57-76. https://doi.org/10.1016/0022-0531(86)90007-4

Guo, H., \& Yannelis, N. C. (2021). Full Implementation under Ambiguity. American Economic Journal: Microeconomics, 13, 148-178.

https://doi.org/10.1257/mic.20180184

Hiriart-Urruty, J. B., \& Lemarechal, C. (2004). Fundamentals of Convex Analysis. New York, NY: Springer-Verlag.

Nishimura, K. G., \& Ozaki, H. (2004). Search and Knightian Uncertainty. Journal of Economic Theory, 119, 299-333. https://doi.org/10.1016/j.jet.2003.04.001

Nishimura, K. G., \& Ozaki, H. (2007). Irreversible Investment and Knightian Uncertainty. Journal of Economic Theory, 136, 668-694. https://doi.org/10.1016/j.jet.2006.10.011

Ohtaki, E. (2011). A Note on the Existence of Monetary Equilibrium in a Stochastic OLG Model with a Finite State Space. Economics Bulletin, 31, 485-492.

Ohtaki, E. (2013). Golden Rule Optimality in Stochastic OLG Economies. Mathematical Social Sciences, 65, 60-66. https://doi.org/10.1016/j.mathsocsci.2012.06.002

Ohtaki, E. (2015). A Note on Existence and Uniqueness of Stationary Monetary Equilibrium in a Stochastic OLG Model. Macroeconomic Dynamics, 19, 701-707.

https://doi.org/10.1017/S1365100513000515 
Ohtaki, E., \& Ozaki, H. (2015). Monetary Equilibria and Knightian Uncertainty. Economic Theory, 59, 435-459. https://doi.org/10.1007/s00199-015-0887-6

Rigotti, L., \& Shannon, C. (2012). Sharing Risk and Ambiguity. Journal of Economic Theory, 147, 2028-2039. https://doi.org/10.1016/j.jet.2012.05.009

Rockafellar, R. T. (1970). Convex Analysis (PMS-28). Princeton, NJ: Princeton University Press. https://doi.org/10.1515/9781400873173

Savage, L. J. (1954) The Foundations of Statistics. New York, NY: John Wiley and Sons.

Schmeidler, D. (1989). Subjective Probability and Expected Utility without Additivity. Econometrica, 57, 571-587. https://doi.org/10.2307/1911053

Wallace, N. (1980). The Overlapping Generations Model of Fiat Money. In J. H. Kareken, \& N. Wallace (Eds.), Models of Monetary Economies (pp. 49-82). Minneapolis, MN: Federal Reserve Bank of Minneapolis. 


\section{Appendix: Proof of Main Results}

In order to obtain our results, we introduce some notation. In the current setting, some of objective functions derived from the utility function $U$ is not necessarily differentiable. However, we can define the "superdifferential" of those functions. The superdifferential of a concave function $f: \operatorname{dom} f \subset \mathfrak{R}^{n} \rightarrow \mathfrak{R}$ at $x \in \operatorname{dom} f$ is defined by

$$
\partial f(x):=\left\{v \in \mathfrak{R}^{n} \mid(\forall y \in \operatorname{dom} f) f(y) \leq f(x)+\langle v, y-x\rangle\right\}
$$

and each of its elements is called a supergradient of $f$ at $x^{9}$. One can immediately show that $\partial f(x)$ is closed and convex. It also follows that $\partial f(x)$ is nonempty and bounded, provided that $x \gg 0^{10}$. Therefore, $\partial f(x)$ is nonempty, compact, and convex for each $x \gg 0$. Furthermore, one can easily observe that all of coordinates of each $v \in \partial f(x)$ are positive when $f$ is strongly monotone.

Proof of Proposition 1. Let $q^{e}$ be a mapping of $\Sigma$ to $\left(0, w^{1}\right)$ and, for each $\sigma \in \Sigma$, define the function $\varphi_{\sigma}: \mathfrak{R} \rightarrow \mathfrak{R}$ by

$$
\varphi_{\sigma(m)}:=v_{1}\left(w^{1}-\frac{q^{e}(\sigma)}{M(\sigma)} m\right)+\min _{\pi \in \mathcal{P}} \sum_{z^{\prime} \in Z} v_{2}\left(w^{2}+\frac{q^{e}\left(\sigma, z^{\prime}\right)}{M\left(\sigma, z^{\prime}\right)}\left(m+T\left(\sigma, z^{\prime}\right)\right)\right) \pi_{z^{\prime}}
$$

for each $m \in \mathfrak{R}$. By the definition, $q^{e}$ is a monetary equilibrium if and only if there exists some stochastic process $m: \Sigma \rightarrow \Re$ such that, for each $\sigma \in \Sigma$, $m^{*}=m(\sigma)$ belongs to the set

$$
\mathcal{M}_{\sigma}:=\arg \max _{m \in \Re} \varphi_{\sigma}(m)
$$

and satisfies that $m^{*}=M(\sigma)$. Given $\sigma \in \Sigma$, it follows from Hiriart-Urruty and Lemaréchal (2004: Corollary D.4.4.4, p. 191) that $m^{*} \in \mathcal{M}_{\sigma}$ if and only if $0 \in \varphi_{\sigma}\left(m^{*}\right)$, which is equivalent to

$$
\begin{aligned}
0 \in & \left\{-\frac{q^{e}(\sigma)}{M(\sigma)} v_{1}^{\prime}\left(w^{1}-\frac{q^{e}(\sigma)}{M(\sigma)} m^{*}\right)\right. \\
& \left.+\sum_{z^{\prime} \in Z} \frac{q^{e}\left(\sigma, z^{\prime}\right)}{M\left(\sigma, z^{\prime}\right)} v_{2}^{\prime}\left(w^{2}+\frac{q^{e}\left(\sigma, z^{\prime}\right)}{M\left(\sigma, z^{\prime}\right)}\left(m^{*}+T\left(\sigma, z^{\prime}\right)\right)\right) \pi_{z^{\prime}} \mid \pi \in \mathcal{B}_{\sigma}^{*}\right\}
\end{aligned}
$$

where

$$
\mathcal{B}_{\sigma}^{*}:=\underset{m \in \Re}{\arg \min } \sum_{z^{\prime} \in Z} v_{2}\left(w^{2}+\frac{q^{e}\left(\sigma, z^{\prime}\right)}{M\left(\sigma, z^{\prime}\right)}\left(m^{*}+T\left(\sigma, z^{\prime}\right)\right)\right) \pi_{z^{\prime}}
$$

By combining this with the condition that $m^{*}=M(\sigma)$ for each $\sigma \in \Sigma$, we can conclude that $q^{e}$ is a monetary equilibrium if and only if

$$
0 \in\left\{-q^{e}(\sigma) v_{1}^{\prime}\left(w^{1}-q^{e}(\sigma)\right)+\sum_{z^{\prime} \in Z} \frac{1}{z^{\prime}} q^{e}\left(\sigma, z^{\prime}\right) v_{2}^{\prime}\left(w^{2}+q^{e}\left(\sigma, z^{\prime}\right)\right) \pi_{z^{\prime}} \mid \pi \in \mathcal{B}_{\sigma}\right\}
$$

for each $\sigma \in \Sigma$, where

${ }^{9}$ For each $x, y \in \mathfrak{R}^{n},\langle x, y\rangle$ represents their inner product, i.e., $\langle x, y\rangle:=x_{1} y_{1}+\cdots+x_{n} y_{n}$, where $x=\left(x_{1}, \cdots, x_{n}\right)$ and $y=\left(y_{1}, \cdots, y_{n}\right)$.

${ }^{10}$ See Rockafellar (1970: Theorem 23.4, p.217) for example. 


$$
\mathcal{B}_{\sigma}:=\underset{m \in \Re}{\arg \min } \sum_{z^{\prime} \in Z} v_{2}\left(w^{2}+q^{e}\left(\sigma, z^{\prime}\right)\right) \pi_{z^{\prime}}
$$

This establishes the proof of Proposition 1.

In order to prove Proposition 2, we prepare a lemma.

Lemma 1. Let $\bar{q}(\zeta)$ be a solution of the equation that $v_{1}^{\prime}\left(w^{1}-q\right)=\zeta v_{2}^{\prime}\left(w^{2}+q\right)$ with respect to $q$. If $\zeta>v_{1}^{\prime}\left(w^{1}\right) / v_{2}^{\prime}\left(w^{2}\right), \bar{q}(\zeta)$ is well-defined and increasing in $\zeta$. Furthermore, it satisfies that $0<\bar{q}(\zeta)<w^{1}$.

Proof of Lemma 1. Given a positive number $x$ such that $x>v_{1}^{\prime}\left(w^{1}\right) / v_{2}^{\prime}\left(w^{2}\right)$, define the function $f_{x}:\left(0, w^{1}\right) \rightarrow \Re$ by

$$
\left(\forall q \in\left(0, w^{1}\right)\right) f_{x}(q):=-v_{1}^{\prime}\left(w^{1}-q\right)+x v_{2}^{\prime}\left(w^{2}+q\right) .
$$

Since $f_{x}$ is continuous, $\lim _{q \downarrow 0} f_{x}(q)=-v_{1}^{\prime}\left(w^{1}\right)+x v_{2}^{\prime}\left(w^{2}\right)>0$, and $\lim _{q \uparrow w^{1}} f_{x}(q)=-\infty<0$, there exists some $\bar{q}(x) \in\left(0, w^{1}\right)$ such that $f_{x}(\bar{q}(x))=0$.

We claim that this $\bar{q}(x)$ is a unique solution of the equation $f_{x}(q)=0$. To verify this, suppose that there exists another solution $\tilde{q}(x) \in\left(0, w^{1}\right)$. Without loss of generality, we assume that $\bar{q}(x)>\tilde{q}(x)$. Then, it follows from strict concavity of $v_{1}$ and $v_{2}$ that

$$
\begin{aligned}
v_{1}^{\prime}\left(w^{1}-\bar{q}(x)\right) & =x v_{2}^{\prime}\left(w^{2}+\bar{q}(x)\right) \\
& <x v_{2}^{\prime}\left(w^{2}+\tilde{q}(x)\right) \\
& =v_{1}^{\prime}\left(w^{1}-\tilde{q}(x)\right) \\
& <v_{1}^{\prime}\left(w^{1}-\bar{q}(x)\right),
\end{aligned}
$$

a contradiction. Therefore, $\bar{q}(x)$ is a unique solution of the equation $f_{x}(q)=0$.

We then claim that $\bar{q}(\cdot)$ is increasing. To verify this, let $g(x):=f(\bar{q}(x))$, which is equal to 0 , provided that $x>v_{1}^{\prime}\left(w^{1}\right) / v_{2}^{\prime}\left(w^{2}\right)$. For each $x$ such that $x>v_{1}^{\prime}\left(w^{1}\right) / v_{2}^{\prime}\left(w^{2}\right)$, it follows that

$0=g^{\prime}(x)=\bar{q}^{\prime}(x) v_{1}^{\prime \prime}\left(w^{1}-\bar{q}(x)\right)+v_{2}^{\prime}\left(w^{2}+\bar{q}(x)\right)+x \bar{q}^{\prime}(x) v_{1}^{\prime \prime}\left(w^{2}+\bar{q}(x)\right), \quad$ which implies that

$$
\bar{q}^{\prime}(x)=-\frac{v_{2}^{\prime}\left(w^{2}+\bar{q}(x)\right)}{v_{1}^{\prime \prime}\left(w^{1}-\bar{q}(x)\right)+x v_{2}^{\prime \prime}\left(w^{2}+\bar{q}(x)\right)}>0 .
$$

This establishes the proof of Lemma 1.

Q.E.D.

Proof of Proposition 2. Let $\zeta \in[\underline{\zeta}, \bar{\zeta}]$ and $\zeta>v_{1}^{\prime}\left(w^{1}\right) / v_{2}^{\prime}\left(w^{2}\right)$. By Lemma $1, \bar{q}(\zeta)$ and $\bar{q}(\bar{\zeta})$ are well-defined and satisfy that $\bar{q}(\zeta) \leq \bar{q}(\bar{\zeta})$. Define the function $h:\left(0, w^{1}\right) \rightarrow \Re$ by

$$
\left(\forall q \in\left(0, w^{1}\right)\right) h(q):=\frac{v_{1}^{\prime}\left(w^{1}-q\right)}{v_{2}^{\prime}\left(w^{2}+q\right)}
$$

Then, it follows from strict concavity of $v_{1}$ and $v_{2}$ that

$$
\left(\forall q \in\left(0, w^{1}\right)\right) h^{\prime}(q)=-v_{1}^{\prime \prime}\left(w^{1}-a\right) v_{2}^{\prime}\left(w^{2}+q\right)-\frac{v_{1}^{\prime}\left(w^{1}-q\right) v_{2}^{\prime \prime}\left(w^{2}+q\right)}{\left(v_{2}^{\prime}\left(w^{2}+q\right)\right)^{2}}>0
$$


Therefore, we have

$$
(\forall q \in \bar{Q}(\zeta)) h(\bar{q}(\zeta)) \leq h(q) \leq h(\bar{q}(\bar{\zeta}))
$$

which implies that

$$
(\forall q \in \bar{Q}(\zeta)) \underline{\zeta} \leq \zeta \leq \frac{v_{1}^{\prime}\left(w^{1}-q\right)}{v_{2}^{\prime}\left(w^{2}+q\right)} \leq \bar{\zeta} .
$$

Now, it is obvious that $\bar{q}^{\text {se }} \in \bar{Q}(\zeta)$ is a deterministic monetary equilibrium.

Q.E.D.

Proof of Proposition 3. First, note that the golden rule optimal allocation $\left(c^{* 1}, c^{* 2}\right)$ is completely characterized by $c^{* 1}+c^{* 2}=w^{1}+w^{2}$ and $v_{1}^{\prime}\left(c^{* 1}\right) / v_{2}^{\prime}\left(c^{* 2}\right)=1$.

Suppose now that $v_{1}^{\prime}\left(w^{1}\right) / v_{2}^{\prime}\left(w^{2}\right)<1$ and $\underline{\zeta} \leq 1 \leq \bar{\zeta}$. Because $v_{1}^{\prime}\left(w^{1}\right) / v_{2}^{\prime}\left(w^{2}\right)<1$, we can immediately find a unique $\bar{q}>0$ such that $v_{1}^{\prime}\left(w^{1}-\bar{q}\right)=v_{2}^{\prime}\left(w^{2}+\bar{q}\right)$. It is easy to verify that this $\bar{q}$ is a desired stationary deterministic monetary equilibrium.

On the other hand, if there is a golden rule optimal stationary deterministic monetary equilibrium $\hat{q}$, it must satisfy that $v_{1}^{\prime}\left(w^{1}-\hat{q}\right)=v_{2}^{\prime}\left(w^{2}+\hat{q}\right)$ by optimality and

$$
v_{2}^{\prime}\left(w^{2}+\hat{q}\right) \underline{\zeta} \leq v_{1}^{\prime}\left(w^{1}-\hat{q}\right) \leq v_{2}^{\prime}\left(w^{2}+\hat{q}\right) \bar{\zeta}
$$

because it is a stationary deterministic monetary equilibrium. Therefore, we obtain that $\underline{\zeta} \leq 1 \leq \bar{\zeta}$.

Uniqueness of a stationary deterministic monetary equilibrium, of which allocation is golden rule optimal follows from uniqueness of $\bar{q}$.

Q.E.D.

Before proving Propositions 4 and 5, we prepare a lemma.

Lemma 2. Let $V_{1}(q):=q v_{1}^{\prime}\left(w^{1}-q\right)$ for all $q \in\left[0, w^{1}\right)$ and $V_{2}(q):=q v_{2}^{\prime}\left(w^{2}+q\right)$ for all $q \in \mathfrak{R}$. Then, $V_{1}$ is increasing and $V_{2}$ is nonincreasing if $-q v_{2}^{\prime \prime}\left(w^{2}+q\right) / v_{2}^{\prime}\left(w^{2}+q\right) \geq 1$ and nondecreasing if $-q v_{2}^{\prime \prime}\left(w^{2}+q\right) / v_{2}^{\prime}\left(w^{2}+q\right) \leq 1$.

Proof of Lemma 2. Since $V_{1}^{\prime}(q)=v_{1}^{\prime}\left(w^{1}-q\right)-q v_{1}^{\prime \prime}\left(w^{1}-q\right)>0, V_{1}$ is increasing on the interior of its domain. On the other hand, since $V_{2}^{\prime}(q)=v_{2}^{\prime}\left(w^{2}+q\right)+q v_{2}^{\prime \prime}\left(w^{2}+q\right), \quad V_{2}$ is nonincreasing if $-q v_{2}^{\prime \prime}\left(w^{2}+q\right) / v_{2}^{\prime}\left(w^{2}+q\right) \geq 1$ and nondecreasing if $-q v_{2}^{\prime \prime}\left(w^{2}+q\right) / v_{2}^{\prime}\left(w^{2}+q\right) \leq 1$.

Proof of Proposition 4. Let $\left(q_{t}\right)_{t=1}^{\infty}$ be a sequence such that $q_{t} \in \bar{Q}^{*}$ for all $t \geq 1$. Then, it follows that, for all $t \geq 1$,

$$
\begin{aligned}
q_{t+1} v_{2}^{\prime}\left(w^{2}+q_{t+1}\right) \underline{\zeta} & \leq \bar{q}_{*} v_{2}^{\prime}\left(w^{2}+\bar{q}_{*}\right) \underline{\zeta} \\
& \leq \bar{q}_{*} v_{1}^{\prime}\left(w^{1}-\bar{q}_{*}\right) \\
& \leq q_{t} v_{1}^{\prime}\left(w^{1}-q_{t}\right) \\
& \leq \bar{q}^{*} v_{1}^{\prime}\left(w^{1}-\bar{q}^{*}\right) \\
& \leq \bar{q}^{*} v_{2}^{\prime}\left(w^{2}+\bar{q}^{*}\right) \bar{\zeta} \\
& \leq q_{t+1} v_{2}^{\prime}\left(w^{2}+q_{t+1}\right) \bar{\zeta}
\end{aligned}
$$


where the first inequality follows from the fact that $V_{2}$ is nonincreasing, the second equality follows from the definition of $\bar{q}_{*}$, the third and fourth inequalities follow from the facts that $q_{t} \in \bar{Q}^{*}$ and that $V_{1}$ is increasing, the fifth equality follows from the definition of $\bar{q}^{*}$, and the last inequality follows from the fact that $V_{2}$ is nonincreasing. Summarizing this result, we have

$$
q_{t+1} v_{2}^{\prime}\left(w^{2}+q_{t+1}\right) \underline{\zeta} \leq q_{t} v_{1}^{\prime}\left(w^{1}-q_{t}\right) \leq q_{t+1} v_{2}^{\prime}\left(w^{2}+q_{t+1}\right) \bar{\zeta} .
$$

By Equation (2), this implies that $\left(q_{t}\right)_{t=1}^{\infty}$ is a deterministic monetary equilibrium. This establishes the proof of Proposition 4.

Q.E.D.

In order to prove Proposition 5, we add two lemmas.

Lemma 3. Both $\bar{q}^{* *}$ and $\bar{q}_{* *}$ defined in Proposition 4 are well-defined and satisfies that $\bar{q}_{*} \leq \bar{q}^{* *}$ and $\bar{q}_{* *} \leq \bar{q}^{*}$.

Proof of Lemma 3. Recall the function $V_{1}$ defined in Lemma 2. It follows from assumptions on $v_{1}$ that $V_{1}^{\prime}(q)=v_{1}^{\prime}\left(w^{1}-q\right)-q v_{1}^{\prime \prime}\left(w^{1}-q\right)>0$ and that $V_{1}(0)=0$ and $\lim _{q \uparrow w^{1}} V_{1}(q)=\infty$. Then, existence and uniqueness of $\bar{q}^{* *}$ and $\bar{q}_{* *}$ follow immediately.

Suppose now that $\bar{q}_{*}>\bar{q}^{* *}$. Then, we have

$$
\begin{aligned}
\bar{q}_{*} v_{1}^{\prime}\left(w^{1}-\bar{q}_{*}\right) & >\bar{q}^{* *} v_{1}^{\prime}\left(w^{1}-\bar{q}^{* *}\right) \\
& =\bar{q}_{*} v_{2}^{\prime}\left(w^{2}+\bar{q}_{*}\right) \bar{\zeta} \\
& >\bar{q}_{*} v_{2}^{\prime}\left(w^{2}+\bar{q}_{*}\right) \underline{\zeta} \\
& =\bar{q}_{*} v_{1}^{\prime}\left(w^{1}-\bar{q}_{*}\right)
\end{aligned}
$$

where the first inequality follows from the fact that $V_{1}$ is increasing, the second equality follows from the definition of $\bar{q}^{* *}$, the third inequality follows from the fact that $\underline{\zeta}<\bar{\zeta}$, and the last equality follows from the definition of $\bar{q}_{*}$. This is, however, a contradiction. Therefore $\bar{q}_{*} \leq \bar{q}^{* *}$.

On the other hand, suppose that $\bar{q}_{* *}>\bar{q}^{*}$. Then, we have

$$
\begin{aligned}
\bar{q}^{*} v_{1}^{\prime}\left(w^{1}-\bar{q}^{*}\right) & <\bar{q}_{* *} v_{1}^{\prime}\left(w^{1}-\bar{q}_{* *}\right) \\
& =\bar{q}^{*} v_{2}^{\prime}\left(w^{2}+\bar{q}^{*}\right) \underline{\zeta} \\
& <\bar{q}^{*} v_{2}^{\prime}\left(w^{2}+\bar{q}^{*}\right) \bar{\zeta} \\
& =\bar{q}^{*} v_{1}^{\prime}\left(w^{1}-\bar{q}^{*}\right)
\end{aligned}
$$

where the first inequality follows from the fact that $V_{1}$ is increasing, the second equality follows from the definition of $\bar{q}_{* *}$, the third inequality follows from the fact that $\underline{\zeta}<\bar{\zeta}$, and the last equality follows from the definition of $\bar{q}^{*}$. This is, however, a contradiction. Therefore $\bar{q}_{* *} \leq \bar{q}^{*}$. This completes the proof of Lemma 3.

Q.E.D.

Lemma 4. If $-q v_{2}^{\prime \prime}\left(w^{2}+q\right) / v_{2}^{\prime}\left(w^{2}+q\right) \leq 1$ for all $q \in \bar{Q}^{*}$, then $\bar{q}_{* *}, \bar{q}^{* *} \in \bar{Q}^{*}$.

Proof of Lemma 4. The fact that $\bar{q}_{*} \leq \bar{q}^{* *}$ and $\bar{q}_{* *} \leq \bar{q}^{*}$ follows from Lemma 3. Hence, we should show that $\bar{q}_{*} \leq \bar{q}_{* *}$ and $\bar{q}^{* *} \leq \bar{q}^{*}$. Recall the functions $V_{1}$ and $V_{2}$ defined in Lemma 2. Then, $V_{1}$ is increasing on its domain and $V_{2}$ is 
nondecreasing on $\bar{Q}^{*}$.

Suppose now that $\bar{q}^{* *}>\bar{q}^{*}$. Then, we have

$$
\begin{aligned}
\bar{q}^{*} v_{1}^{\prime}\left(w^{1}-\bar{q}^{*}\right) & <\bar{q}^{* *} v_{1}^{\prime}\left(w-\bar{q}^{* *}\right) \\
& =\bar{q}_{*} v_{2}^{\prime}\left(w^{2}+\bar{q}_{*}\right) \bar{\zeta} \\
& \leq \bar{q}^{*} v_{2}^{\prime}\left(w^{2}+\bar{q}^{*}\right) \bar{\zeta} \\
& =\bar{q}^{*} v_{1}^{\prime}\left(w^{1}-\bar{q}^{*}\right)
\end{aligned}
$$

where the first inequality follows from the fact that $V_{1}$ is increasing, the second equality follows from the definition of $\bar{q}^{* *}$, the third inequality follows from the fact that $V_{2}$ is nondecreasing, and the last equality follows from the definition of $\bar{q}^{*}$. This is, however, a contradiction. Therefore, $\bar{q}^{* *} \leq \bar{q}^{*}$.

On the other hand, suppose that $\bar{q}_{*}>\bar{q}_{* *}$. Then, we have

$$
\begin{aligned}
\bar{q}_{*} v_{1}^{\prime}\left(w^{1}-\bar{q}_{*}\right) & >\bar{q}_{* *} v_{1}^{\prime}\left(w-\bar{q}_{* *}\right) \\
& =\bar{q}^{*} v_{2}^{\prime}\left(w^{2}+\bar{q}^{*}\right) \underline{\zeta} \\
& \geq \bar{q}_{*} v_{2}^{\prime}\left(w^{2}+\bar{q}_{*}\right) \underline{\zeta} \\
& =\bar{q}_{*} v_{1}^{\prime}\left(w^{1}-\bar{q}_{*}\right),
\end{aligned}
$$

where the first inequality follows from the fact that $V_{1}$ is increasing, the second equality follows from the definition of $\bar{q}_{* *}$, the third inequality follows from the fact that $V_{2}$ is nondecreasing, and the last equality follows from the definition of $\bar{q}_{*}$. This is, however, a contradiction. Therefore $\bar{q}_{*} \leq \bar{q}_{* *}$. This completes the proof of Lemma 4.

Q.E.D.

Proof of Proposition 5. Recall the functions $V_{1}$ and $V_{2}$ defined in Lemma 2. Under assumptions in this proposition, $V_{1}$ is increasing on its domain and $V_{2}$ is nondecreasing on $\bar{Q}^{*}$.

By Lemma $4, \bar{q}_{* *}, \bar{q}^{* *} \in \bar{Q}^{*}$. Note that Equation (3) is equivalent to $\bar{q}_{* *} v_{1}^{\prime}\left(w^{1}-\bar{q}_{* *}\right)<\bar{q}^{* *} v_{1}^{\prime}\left(w^{1}-\bar{q}^{* *}\right)$ by the definitions of $\bar{q}_{* *}$ and $\bar{q}^{* *}$. This implies that $\bar{q}_{* *}<\bar{q}^{* *}$, since $V_{1}$ is increasing.

Let $\left(q_{t}\right)_{t=1}^{\infty}$ be a sequence such that $q_{t} \in\left[\bar{q}_{* *}, \bar{q}^{* *}\right]$ for all $t \geq 0$. Then, it follows that, for all $t \geq 0$,

$$
\begin{aligned}
q_{t+1} v_{2}^{\prime}\left(w^{2}+q_{t+1}\right) \underline{\zeta} & \leq \bar{q}^{*} v_{2}^{\prime}\left(w^{2}+\bar{q}^{*}\right) \underline{\zeta} \\
& =\bar{q}_{* *} v_{1}^{\prime}\left(w^{1}-\bar{q}_{* *}\right) \\
& \leq q_{t} v_{1}^{\prime}\left(w^{1}-q_{t}\right) \\
& \leq \bar{q}^{* *} v_{1}^{\prime}\left(w^{1}-\bar{q}^{* *}\right) \\
& =\bar{q}_{*} v_{2}^{\prime}\left(w^{2}+\bar{q}_{*}\right) \bar{\zeta} \\
& \leq q_{t+1} v_{2}^{\prime}\left(w^{2}+q_{t+1}\right) \bar{\zeta},
\end{aligned}
$$

where the first inequality follows from the fact that $V_{2}$ is nondecreasing, the second equality follows from the definition of $\bar{q}_{* *}$, the third and fourth inequalities follows from the facts that $q_{t} \in\left[\bar{q}_{* *}, \bar{q}^{* *}\right]$ and that $V_{1}$ is increasing, the 
fifth equality follows from the definition of $\bar{q}^{* *}$, and the last inequality follows from the fact that $V_{2}$ is nondecreasing. Summarizing this result, we have,

$$
q_{t+1} v_{2}^{\prime}\left(w^{2}+q_{t+1}\right) \underline{\zeta} \leq q_{t} v_{1}^{\prime}\left(w^{1}-q_{t}\right) \leq q_{t+1} v_{2}^{\prime}\left(w^{2}+q_{t+1}\right) \bar{\zeta}
$$

By Equation (2), this implies that $\left(q_{t}\right)_{t=1}^{\infty}$ is a deterministic monetary equilibrium.

This establishes the proof of Proposition 5.

Q.E.D. 